\title{
Performance Evaluation of Direct Sequence WCDMA Power Control
}

\author{
Rasha Ali Mohammed Ahmed ${ }^{1}$, Dr. Haala El Dawoo ${ }^{2}$ \\ Department of Communications, Faculty of Engineering, Al-Néelain University
}

\begin{abstract}
The aim of this paper is a study of Wide Code Division Multiple Access (WCDMA) power control through the Signal-toNoise Ratio against Bit Error Rate. The various factors that took into account for the analysis of performances the Additive White Gaussian (AWGN) noise, fading, multiple path accesses and case No of channel. Then analyze the performance of different factors using Simulation MAT-LAB software program and then get the results in the form of tables and graphs.
\end{abstract}

Keywords: WiMAX, Ber, delay, TCP/IP, Fixed

\section{Introduction}

CDMA was developed by the Allies to resist jamming of the radio signal by the enemy during World War 2. The use of WCDMA for civilian mobile communication did not take place until 40 years later called G2.Additional systems have been used to increase the speed of data transfer on the second generation systems, And has called me G2.5 After the great success of second generation systems began to move towards a single system includes Cellular systems and paging systems and satellite systems and integrate them in a unified global system called Wideband CDMA is a thirdgeneration $(3 \mathrm{G})$ Wireless.

\section{Methodology}

The block diagram is shown in the complete system description which shows the steps of the data processing, transmitting and receiving. The (Data) block in the diagram represent the data source (data to be sent). After the data have been generated the (BPSK Mod) implement the process in which data will be modulated with BPSK digital modulation.

After that data will be multiplied by the spreading code the data will be ready to be transmitted over the ( nun channel Multipath and AWGN channel) which represent the channel after adding multipath and AWGN effects to the data in the channel. In the receiver party the data which have been modulated and spread earlier have to be dispread and demodulated. Therefore to perform the dispread process the data will be multiplied by the spreading code (the same code in the transmitter).And then demodulated to abstract the received data, which is going to be compared with the data sent by the transmitter to calculate the bit error rate (BER) in the (BER calculator), and then determine if to increase or decrease the power of transmitter by sending TPC power increase or decrease power by step size.

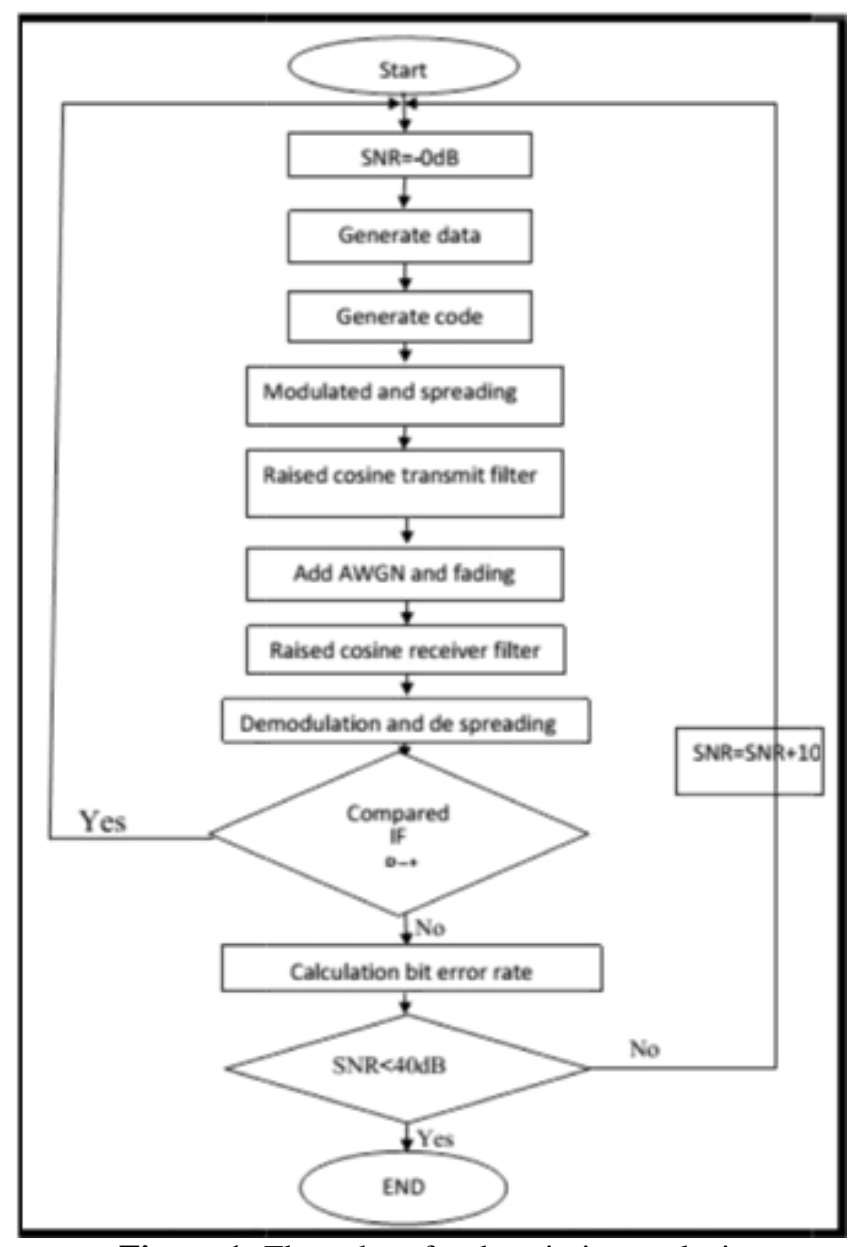

Figure 1: Flow chart for descriptive analysis 
International Journal of Science and Research (IJSR)

ISSN (Online): 2319-7064

Index Copernicus Value (2013): 6.14 | Impact Factor (2015): 6.391

3. Simulator Parameters

Table 1: Simulator parameters

\begin{tabular}{|l|l|}
\hline Parameters & Value \\
\hline Network & WCDMA \\
\hline SNR & $0-40$ \\
\hline Spreading & Wash \\
\hline Filter & RC-Filter \\
\hline Noise & AWGN \\
\hline Fading & Multi path \\
\hline WCDMA & Direct Sequence \\
\hline Encoding data rate & Configuration $1 / 4$ \\
\hline Power control & Nun power control,1,1/2,1/4 \\
\hline Convolution rate & 9 \\
\hline Data r ate & 1.5 \\
\hline Decoder & Encode \\
\hline
\end{tabular}

\section{Simulation Design}

The simulation has been designed using the Mat-lab semi link (block set diagrams), which support the oriented purpose

\section{Selecting Mat-lab}

There are several reasons for selecting mat-lab program: - Can be used to implement hard ware components.

- May work in Windows and Linux environment.

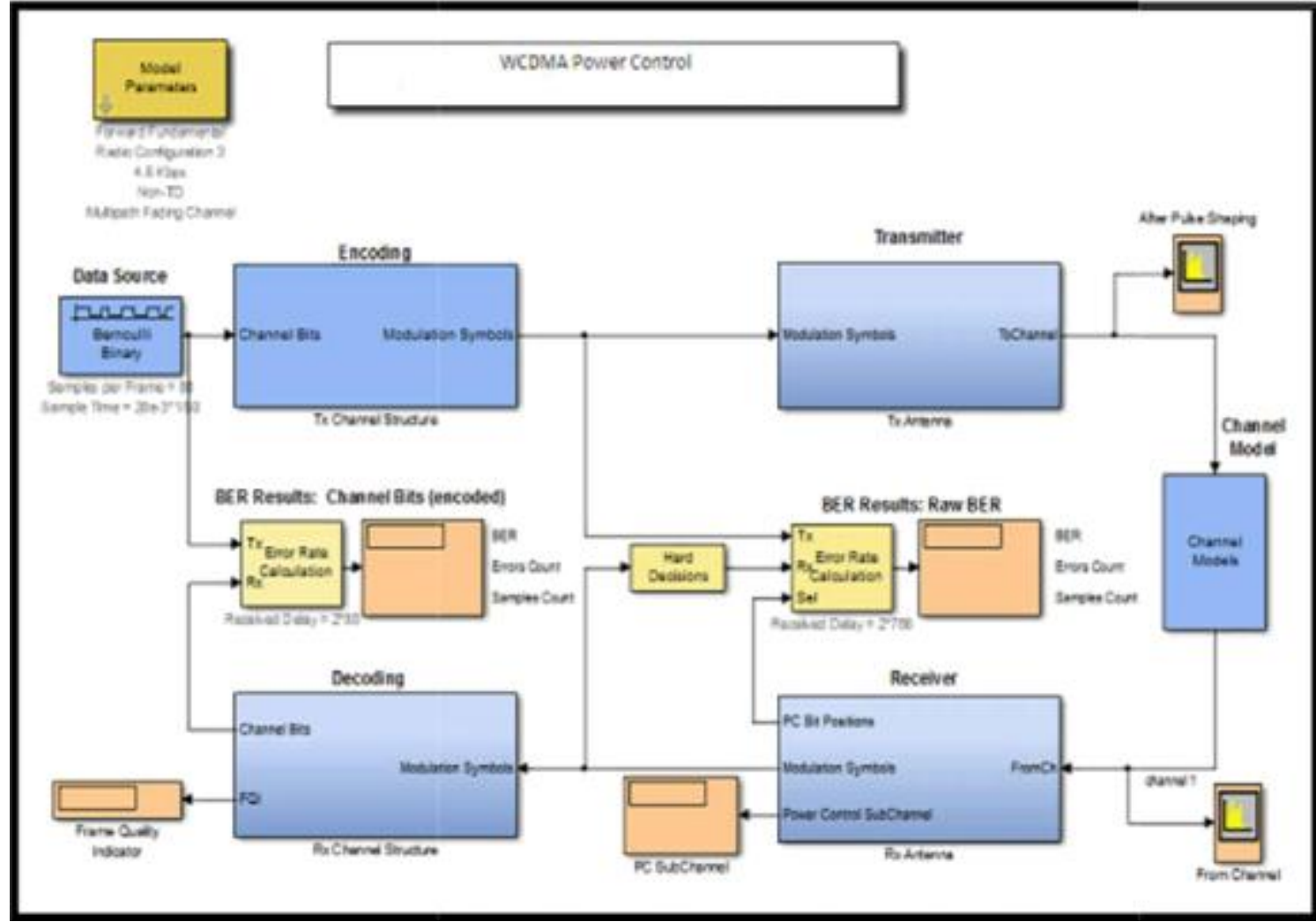

Figure 2: Computer model of power control in wimax

\section{Result}

1) The channel mode is AW GN with different data rate

Volume 5 Issue 6, June 2016 
International Journal of Science and Research (IJSR)

ISSN (Online): 2319-7064

Index Copernicus Value (2013): 6.14 | Impact Factor (2015): 6.391

Table 2: BER vs. SNR within data rate $=1.5 \mathrm{kbps}, 2.7 \mathrm{kbps}, 4.8 \mathrm{kbps}, 9.6 \mathrm{kbps}$ in AWGN, Power control sub channel insertion rate: Non power control

\begin{tabular}{|l|l|l|l|l|}
\hline SNR & $\begin{array}{l}\text { BER within } \\
\text { data rate }=1.5 \mathrm{kbps}\end{array}$ & $\begin{array}{l}\text { BER within } \\
\text { data rate }=2.7 \mathrm{kbps}\end{array}$ & $\begin{array}{l}\text { BER within } \\
\text { data rate }=4.8 \mathrm{kbps}\end{array}$ & $\begin{array}{l}\text { BER within } \\
\text { data rate }=9.6 \mathrm{kbps}\end{array}$ \\
\hline 0 & $7.828 \mathrm{e}-006$ & $1.044 \mathrm{e}-005$ & $1.044 \mathrm{e}-005$ & $2.088 \mathrm{e}-005$ \\
\hline 10 & 0 & 0 & 0 & 0 \\
\hline 20 & 0 & 0 & 0 & 0 \\
\hline 30 & 0 & 0 & 0 & 0 \\
\hline 40 & 0 & 0 & 0 & 0 \\
\hline \hline
\end{tabular}

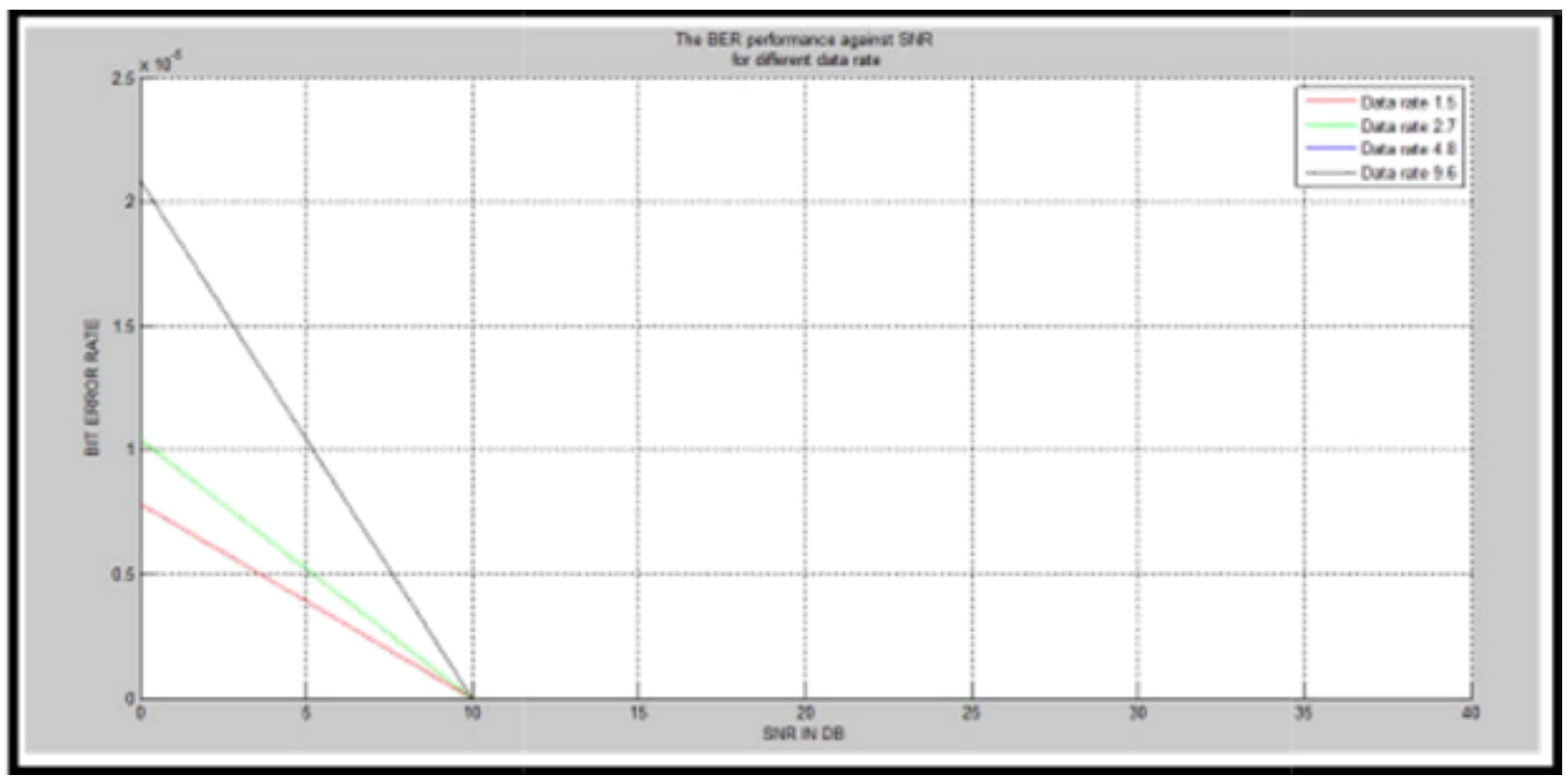

Figure 3: BER vs. SNR within data rate $=1.5 \mathrm{kbps}, 2.7 \mathrm{kbps}, 4.8 \mathrm{kbps}, 9.6 \mathrm{kbps}$ in AWGN, Power control sub channel insertion rate: Non poweer control

2) The maximum Doppler frequency shift is 286 with data rate 1.5

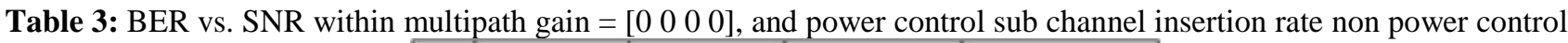

\begin{tabular}{|c|c|c|c|c|}
\hline SNR & $\left.\begin{array}{l}\text { BER with gain } \\
\text { multipath } \\
\text { factor }=\left[\begin{array}{lll}0 & 0 & 0\end{array}\right] \\
\text { o] }\end{array}\right]$ & $\begin{array}{l}\text { BER with gain } \\
\text { multipath } \\
\text { factor= }\left[\begin{array}{lll}0 & 0 & 0\end{array}\right. \\
0\end{array}$ & $\begin{array}{l}\text { BER with gain } \\
\text { multipath } \\
\text { factor= }\left[\begin{array}{llll}0 & 0 & 0 & 0\end{array}\right]\end{array}$ & 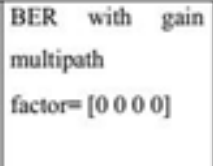 \\
\hline 0 & 0.001086 & 0.01067 & 0.01045 & 0.01086 \\
\hline 5 & 0.001582 & 0.001554 & 0.001553 & 0.001553 \\
\hline 10 & 0.000977 & 0.02192 & 0.0009428 & 0.000977 \\
\hline 15 & 0.001075 & 0.001077 & 0.001089 & 0.001089 \\
\hline 20 & 0.0001086 & 0.01916 & 0.01913 & 0.0001086 \\
\hline 25 & 0.001049 & 0.001039 & 0.001062 & 0.001062 \\
\hline 30 & $2.714 c-05$ & 0.01878 & $2.77 e-05$ & $2.714 c-05$ \\
\hline 35 & 0.001066 & 0.001049 & 0.001062 & 0.001062 \\
\hline 40 & 0 & 0.01841 & 0.1861 & 0 \\
\hline
\end{tabular}

Volume 5 Issue 6, June 2016 
International Journal of Science and Research (IJSR)

ISSN (Online): 2319-7064

Index Copernicus Value (2013): 6.14 | Impact Factor (2015): 6.391

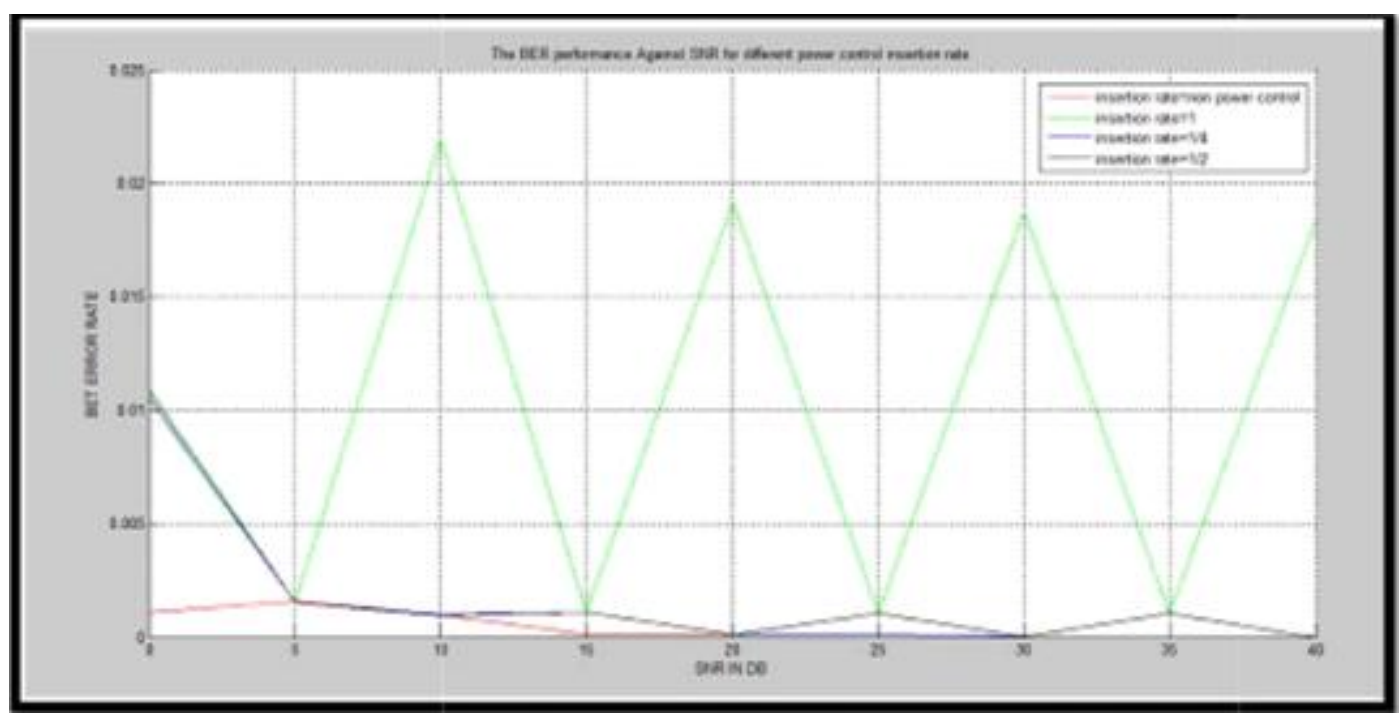

Figure 5: BER vs. SNR $w$ ithin multipath gain $=\left[\begin{array}{lllll}0 & 0 & 0 & 0\end{array}\right]$ and power control sub channel inse rtion rate non power control , 1 , $1 / 2,1 / 4$

3) The maximum Doppler frequency shift is 486 with data rate 1.5

4) The maximum Doppler frequency shift is 786 with data rate 1.5

Table 4: BER vs. SNR within different multipath fading $=[0$ $\left.\begin{array}{lll}0 & 0 & 0\end{array}\right]$ and power control sub channel insertion rate nun

\begin{tabular}{|c|c|c|c|c|}
\hline SNR & $\begin{array}{l}\text { BER with gain } \\
\text { multipath factor } \\
=\left[\begin{array}{llll}0 & 0 & 0 & 0\end{array}\right]\end{array}$ & 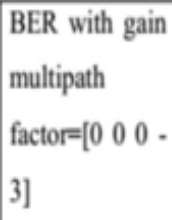 & 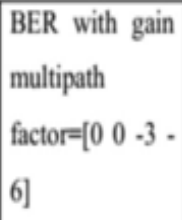 & $\begin{array}{l}\text { BER with gain } \\
\text { multipath } \\
\text { factor }=\left[\begin{array}{llll}0 & -3 & -6 & -9\end{array}\right]\end{array}$ \\
\hline 0 & 0.00272 & 0.002606 & 0.005536 & 0.01198 \\
\hline 5 & 0.0008329 & 0.0007912 & 0.0008278 & 0.001558 \\
\hline 10 & 0.0004058 & 0.0003605 & 0.0006242 & 0.001355 \\
\hline 15 & 0.000251 & 0.0002346 & 0.000251 & 0.00113 \\
\hline
\end{tabular}

\begin{tabular}{|l|l|l|l|l|}
\hline 20 & 0.0002029 & 0.0001941 & 0.0004342 & 0.0004517 \\
\hline 25 & 0.0002168 & 0.0001964 & 0.0002029 & 0.001135 \\
\hline 30 & 0.0002029 & 0.0002218 & 0.0003528 & 0.0003455 \\
\hline 35 & 0.0002111 & 0.000192 & 0.0001923 & 0.00113 \\
\hline 40 & 0.0002029 & 0.0002218 & 0.0003528 & 0.0002657 \\
\hline
\end{tabular}

Volume 5 Issue 6, June 2016 
International Journal of Science and Research (IJSR)

ISSN (Online): 2319-7064

Index Copernicus Value (2013): 6.14 | Impact Factor (2015): 6.391

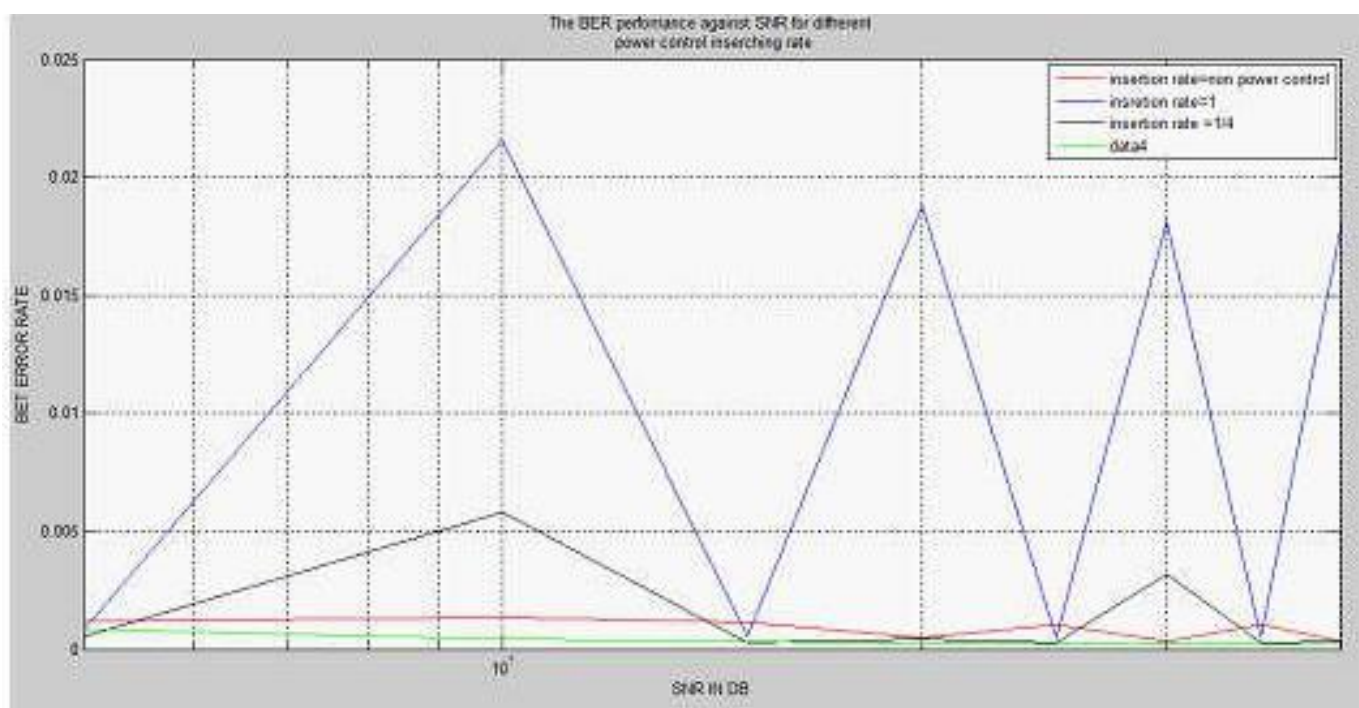

Figure 4: BER vs. SNR within different multipath fading=[ $\left.\begin{array}{llll}0 & 0 & 0 & 0\end{array}\right]$ and power control sub channel insertion rate nun power control $, 1,1 / 2, .1 / 4$

\section{Results Discussion}

Whenever decrease of data rate, the BER where decreased and decrease of Doppler Frequency, the BER where decreased. Data increase of multipath, the BER where decreased.

\section{Conclusion}

The study, analysis, planning, design and simulation of software program to simulate the performance of WCDMA using power control had been done using mat-lab software program.

The parameters which were taken into consideration of the simulation was: Multipath fading, AWGN, modulation and power control rate.

After the execution of the simulation, the results were obtained in term of table and graph. From the result we observed:

- Whenever decrease of data rate, the BER where decreased.

- Whenever decrease of Doppler Frequency, the BER where decreased

- Whenever increase of multipath, the BER where decreased

\section{References}

[1] Keiji Tachikawa, W-CDMA MOBILE COMMUNICATIONS SYSTEM, 2002.

[2] Prasad, R., W Mohr, and W. konhauser, "ThirdGeneration Mobile Communication System", Norwood, MA: Artech House, 2000.

[3] ThatchChlorate and Hyuck M. Kwon."Combination of Power Controls for CDMA 2000 Wireless Communications System. Inprecede".

[4] Wireless Communications Principles and Practice 2nd Edition, T Rappaport, Prentice Hall-2001

[5] John Wiley \& Sons, Adaptive WCDMA: Theory And Practice. Savo G. Glisic2003.

[6] Rachod Patachaianand, Kumbesan Sandrasegaran, Performance Comparison of Adaptive Power Control in UMTS, University of Technology Sydney
[7] I. Forkel, R. Seidenberg, R. Pabst, G. Heidelberger, "Performance Evaluation of Power Control Algorithms in Cellular UTRA Systems," in Proc. Of Second

[8] Loutfi Nuaymi, Xavier Lagrange, Philippe Godlewski, APower Control Algorithm for 3G WCDMA System

[9] Dr. Khalid Hamid Bilal, mobile cellular system International Conference on 3G Mobile.

[10] That Chlorate and Hyuck M. Kwon."Combination of Power Controls for CDMA 2000 Wireless Communications System. Inprecede".

Volume 5 Issue 6, June 2016 